\title{
The safety and serious adverse events of approved ALK inhibitors in malignancies: a meta-analysis
}

This article was published in the following Dove Press journal:

Cancer Management and Research

\section{Helei Hou* \\ Dantong Sun* \\ Kewei Liu \\ Man Jiang \\ Dong Liu \\ Jingjuan Zhu \\ $\mathrm{Na}$ Zhou \\ Jing Cong \\ Xiaochun Zhang}

Department of Medical Oncology, The Affiliated Hospital of Qingdao University, Qingdao University, Qingdao, People's Republic of China

*These authors contributed equally to this work
Correspondence: Xiaochun Zhang Department of Medical Oncology, The Affiliated Hospital of Qingdao University, Qingdao University, 16 jiangsu Road, Qingdao 266003, People's Republic of China

Tel 0860532 829| 327|

Fax 086053282913271

Email zxc9670@qdu.edu.cn
Background: A total of $2 \%-7 \%$ of non-small cell lung cancer (NSCLC) patients have anaplastic lymphoma kinase (ALK) mutations. At present, three or more generations of ALK inhibitors have been used for ALK-positive NSCLC treatment, including crizotinib, alectinib, ceritinib, and brigatinib. Although most adverse events (AEs) of ALK inhibitors are grades 1 to 2 and generally can be well tolerated, serious adverse events (SAEs) of ALK inhibitors lack data analysis, and the lung toxicity of ALK inhibitors needs attention. Thus, we performed this meta-analysis to evaluate the safety of ALK inhibitors, especially in terms of drug-related SAEs.

Methods: A total of 19 studies from 4 databases (PubMed, Science Direct, ClinicalTrials. gov and Cochrane Library) were included in this meta-analysis. All statistical analyses in this meta-analysis were performed with the STATA 14.0 software. We analyzed the incidences of total AEs, total SAEs and SAEs for different ALK inhibitors.

Results: AEs of the ALK inhibitors occurred in almost all participants, and SAEs occurred in more than $20 \%$ of the participants. For ceritinib and brigatinib, SAEs occurred in more than $40 \%$ of the participants. Alectinib is most likely the safest of the two generations of ALK inhibitors. Generally, the ALK inhibitors showed significant lung toxicity.

Conclusion: In conclusion, attention should be focused on ALK inhibitor-related SAEs, especially lung toxicity. According to this meta-analysis, alxectinib seems to be the safest ALK inhibitor. Physicians should focus on the related SAEs when prescribing ALK inhibitors.

Keywords: ALK inhibitors, safety, serious adverse events, lung toxicity

\section{Background}

NSCLC patients with ALK mutations and gene fusion were first described in 2007. ${ }^{1,2}$ A total of $2 \%-7 \%$ of NSCLC patients have ALK mutations or ALK gene fusions. ${ }^{3}$ Patients with ALK mutation have some significant characteristics; for instance, most are young patients with little to no smoking history, and the most common pathological type is adenocarcinoma. ${ }^{4,5}$ Decades later, three or more generations of ALK inhibitors have been used for ALK-positive NSCLC treatment, including crizotinib, alectinib, ceritinib, and brigatinib.

Crizotinib was the first generation small molecule ALK tyrosine kinase inhibitor (TKI) approved by the FDA for ALK-positive NSCLC patients in 2001. Crizotinib was proven to improve progression-free survival (PFS), and patients had a satisfactory objective response rate (ORR) to the drug. ${ }^{6,7}$ However, approximately $73 \%$ of patients acquired resistance after less than 1 year on crizotinib treatment (medium PFS: 10.9 months). ${ }^{7}$ Fortunately, the second and third generation ALK inhibitors have provided crizotinib-resistant patients with more options. 
Although most of AEs of ALK inhibitors are grades 1 to 2 and generally can be well tolerated by patients, clinical data analysis of the SAEs of the two generations of ALK inhibitors is lacking, and the lung toxicity of ALK inhibitors requires attention. ${ }^{8}$ Meanwhile, ALK inhibitorrelated dyspnea and interstitial lung disease (ILD) have been detected during the treatment process. ${ }^{9,10}$ Thus, we performed this meta-analysis to evaluate the safety of two generations of ALK inhibitors, especially in terms of drugrelated SAEs..

\section{Methods}

\section{Search strategy for the studies}

In June 2018, 2 authors (Hou HL and Sun DT) searched four databases independently, including PubMed, Science Direct, ClinicalTrials.gov, and Cochrane Library. MeSH terms for all keywords were used in the search strategies, including crizotinib, PF-06260182, alectinib, CH5424802, ceritinib, LDK378, brigatinib and AP26113. All keywords were searched in the databases separately. Any disagreement concerning whether the study should be included was discussed by all authors. In addition, we contacted some of the corresponding authors of the studies if the databases failed to provide sufficient information.

\section{Literature selection criteria}

All clinical trials evaluating the safety of ALK inhibitors were considered eligible for the analysis. Two authors (Sun DT and Hou HL) completed the literature screening independently. The inclusion criteria were as follows: 1) trial phases: I to III, only in English; 2) participant types: advanced lung cancer patients; 3) intervention types: patients treated with ALK inhibitors; and 4) outcome measure types: the incidences of pooled AEs and SAEs, and the incidences of all types of SAEs.

The exclusion criteria were as follows: case reports, reviews or meta-analyses; duplicate studies; animal or cell experiments and articles not written in English.

\section{Data extraction}

Two authors (Sun DT and Hou HL) completed the related literature data extraction independently, including the study ID, ALK inhibitor types, treatment lines, patients' races, trial phases, cancer types, and driver mutations. The characteristics of the included studies are shown in Table 1. We also extracted the following research indicators selected in this meta-analysis from the included studies: the incidence of total AEs; the incidence of total SAEs and the incidences of common types of SAEs, which are shown in Table S1. Disagreements concerning the data extraction results were discussed by all authors.

\section{Quality assessment}

Two authors (Hou HL and Zhang XC) assessed the quality of the studies independently after reading the full text of each study. We used the Newcastle-Ottawa scale (NOS) to assess the quality of non-randomized controlled trials (non-RCTs) in this meta-analysis. ${ }^{11}$ The quality of the study was considered "poor" if the NOS score was less than 4 . If the score was between 4 and 6 , we considered the quality of the study "moderate". Studies with scores between 7 and 9 were considered "high quality". The NOS scores of the 21 included studies are shown in Table S1, and the details of the quality assessment by NOS are shown in the supplementary files (Table S2). The risk of bias of the randomized controlled trials (RCTs) was independently assessed by the 2 investigators above using the Cochrane collaboration's tool for assessing the risk of bias in RCTs. ${ }^{12}$ The details of the assessment are shown in the supplementary files (Figure S1).

\section{Statistical analysis}

All statistical analyses in this meta-analysis were performed with the STATA 14.0 software (Stata Corp., College Station, TX, USA). The results are expressed as the rate and $95 \%$ confidence interval (CI). In this metaanalysis, we used the random-effects model to perform the statistical analyses and the Chi-square test and $\mathrm{I}^{2}$ statistic to assess inter-study heterogeneity. A $p$-value $>0.1$ and an $\mathrm{I}^{2}<50 \%$ indicate that the heterogeneity is not statistically significant. If the $p$-value was less than 0.1 and $\mathrm{I}^{2}>50 \%$, significant heterogeneity existed among the studies, and subgroup analyses were performed to assess the heterogeneity. We followed the Cochrane handbook, and no post hoc analysis was performed. Begg's and Egger's tests were used to evaluate publication bias in this meta-analysis. The trim and fill method was used for the sensitivity analysis in this meta-analysis.

\section{Results}

Figure 1 describes the literature selection process of the included studies. A total of 13,253 references were identified after database searching (PubMed 7,514, Cochrane Library 347, Science Direct 5,183, and ClinicalTrials.gov 209). A total of 9,096 references remained after duplicates were 


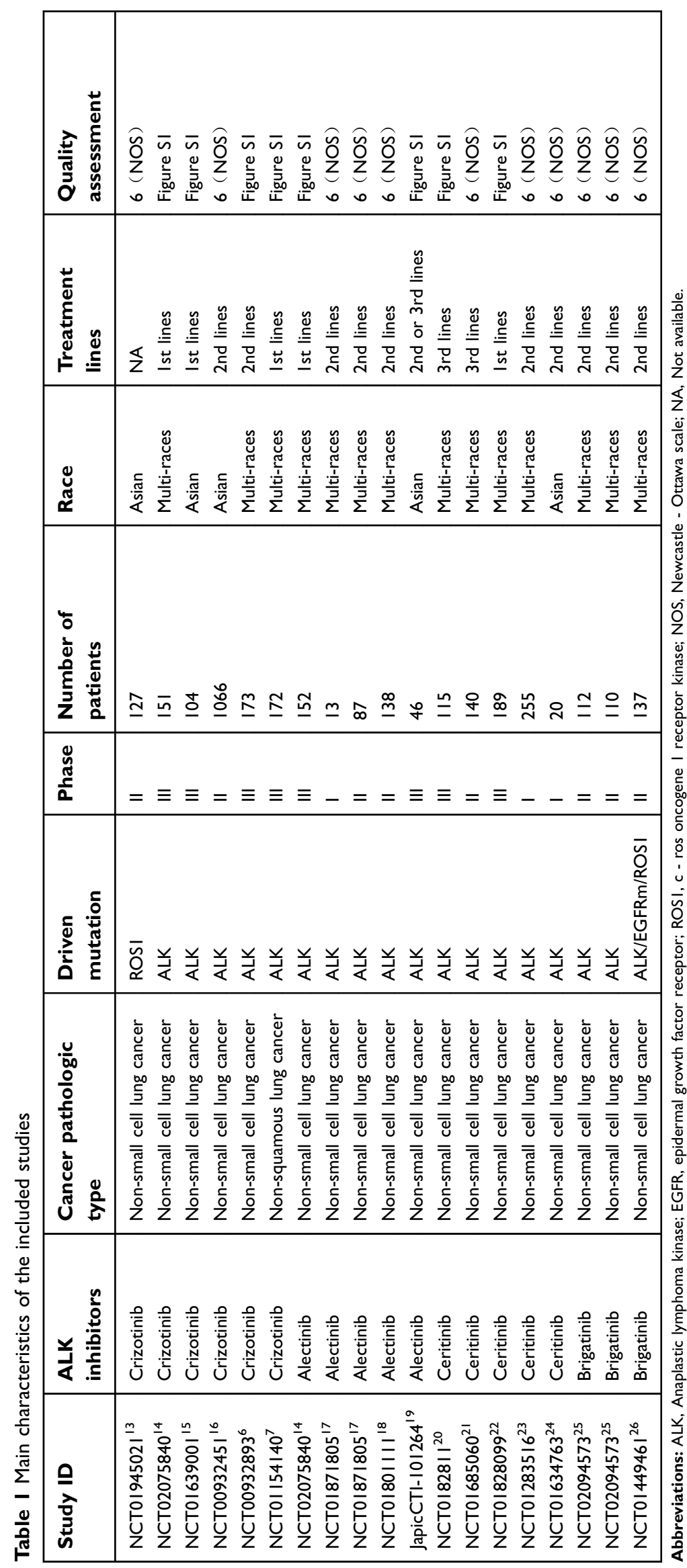




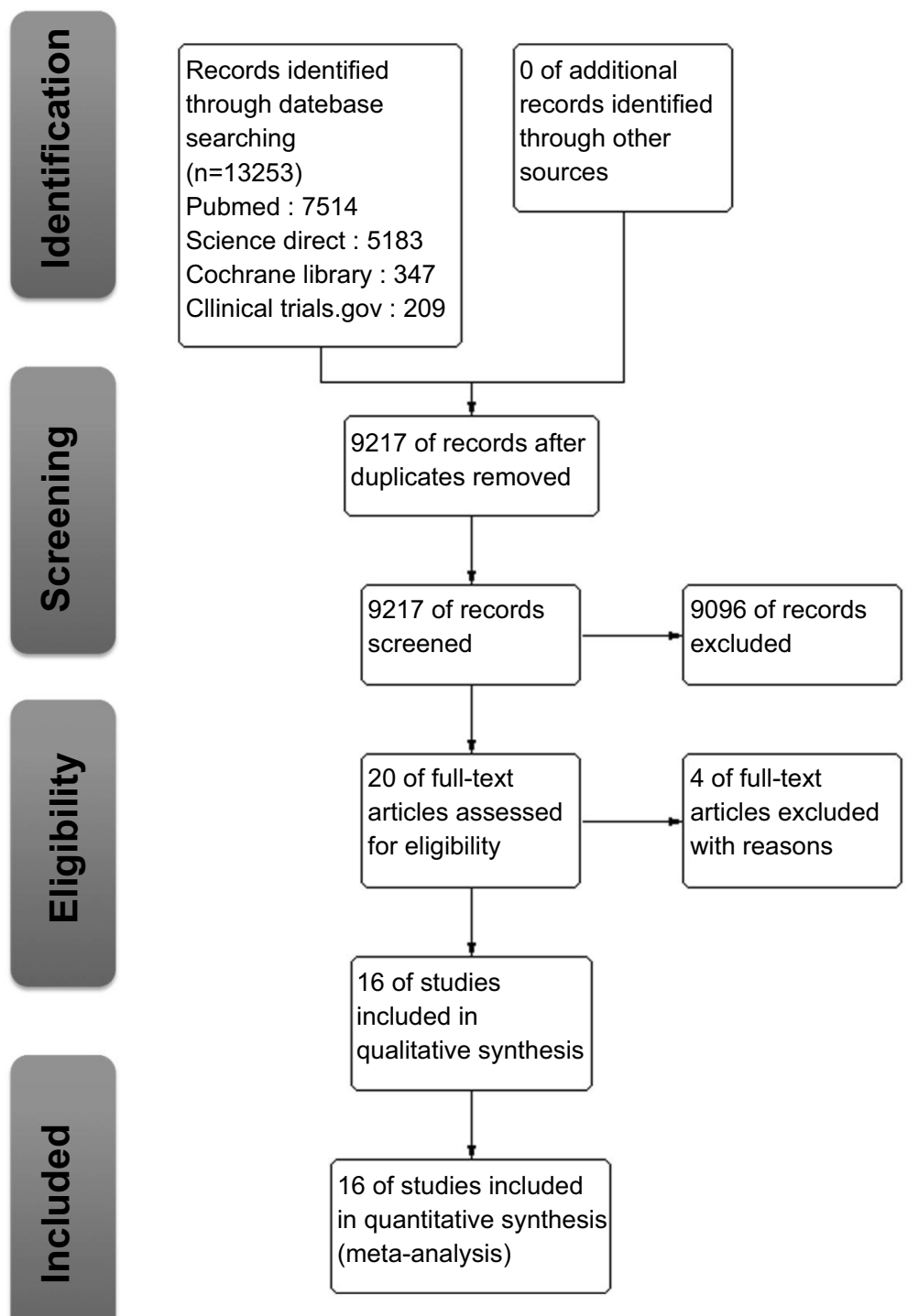

Figure I Flow chart of the article selection process.

removed. A total of 23 references remained after the first screening, and 4 references were excluded for multiple reasons, including 2 that were case-series reports and 2 that were duplicate references. Finally, 19 studies with a total of 3,307 patients were included for the safety evaluation of the two generations of ALK inhibitors.

\section{Results of the pooled AEs and SAEs}

AEs were detected in almost all participants in this metaanalysis. The overall incidences of AEs for the two generations of ALK inhibitors were as follows: crizotinib: $98.27 \%$ (95\% CI: 96.94-99.61); alectinib: 96.24\% (95\% CI: 92.88-99.60); ceritinib: $99.45 \%$ (95\% CI: 98.90-99.99); and brigatinib: 94.77\% (95\% CI: 92.47-97.08). Importantly, the incidences of the SAEs were not as low as expected. The incidences of the SAEs were as follows: crizotinib: $38.09 \% \quad(95 \%$ CI: $26.93-47.26$ ); alectinib: $26.24 \%$ (95\% CI: $14.15-38.33$ ); ceritinib: $41.44 \%$ (95\% CI: 37.15-45.73); and brigatinib: 41.68\% (95\% CI: 36.21-47.14). The incidences of the AEs and SAEs are shown in Figure 2A, and the details of the analysis of the $\mathrm{AE}$ and $\mathrm{SAE}$ incidences are shown in the supplementary files (Figures S2 and S3).

\section{The SAEs of five major systems}

The incidences of SAEs for five major systems are summarized in Figure 2B. All SAEs in the five systems followed the classification from clinicaltrails.gov. The ALK inhibitors showed significant respiratory system toxicity. 


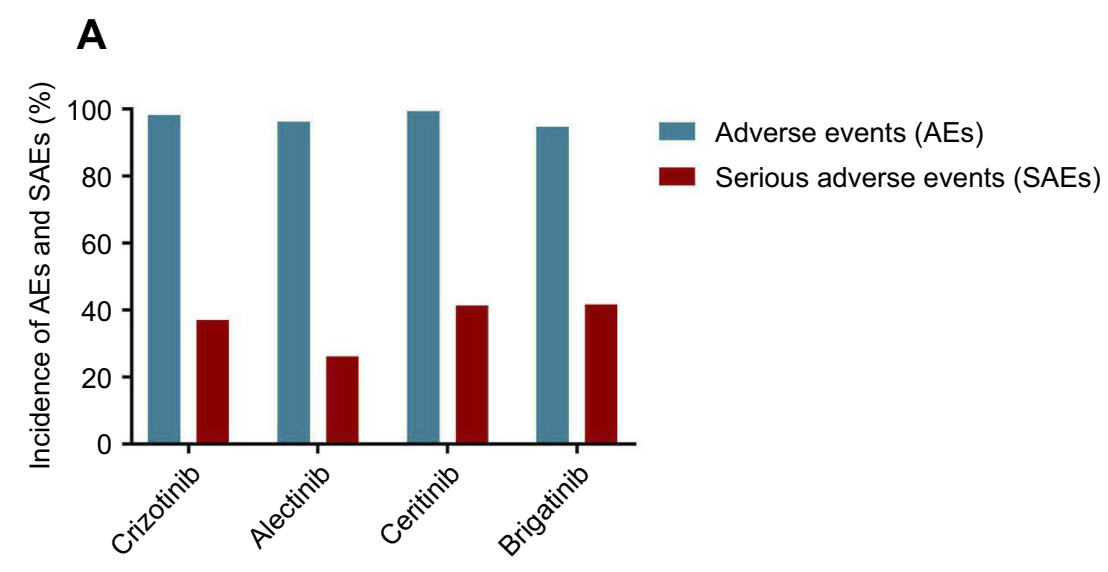

B

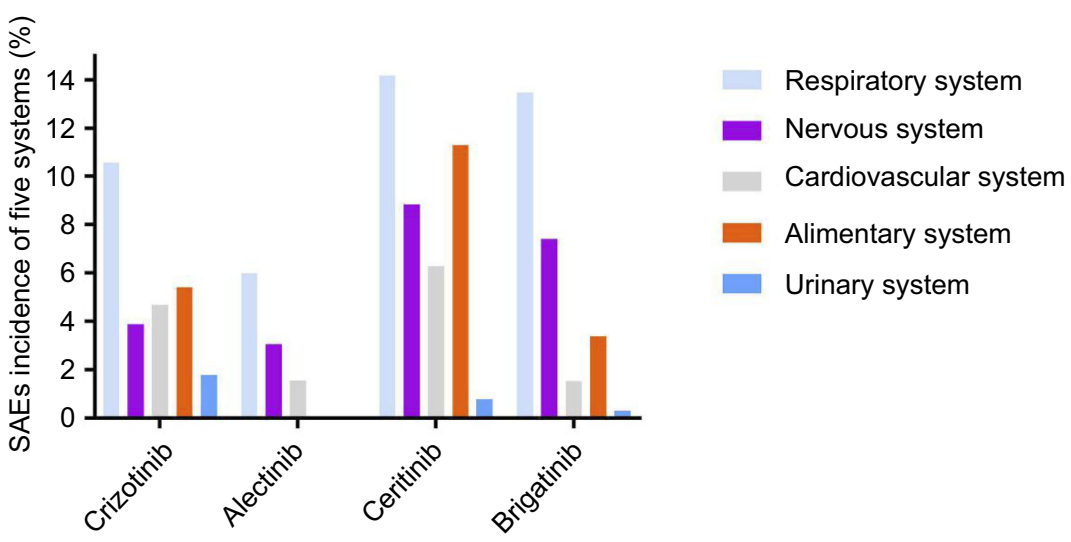

Figure 2. Safety of two generations ALK inhibitors. (A) Incidences of pooled AEs and SAEs. (B) Incidences of SAEs in five systems. Abbreviation: ALK, Anaplastic lymphoma kinase.

The incidences of SAEs in the respiratory system were as follows: crizotinib: $10.57 \%$ (95\% CI: 7.22-13.92); alectinib: $5.98 \%$ (95\% CI: 3.59-8.38); ceritinib: $14.17 \%$ (95\% CI: $10.85-17.48)$; and brigatinib: $13.48 \% \quad(95 \%$ CI: 6.24-20.71). In addition to SAEs in the respiratory system, the most common 3 systemic SAEs were found in the nervous system, cardiovascular system and alimentary system. The first generation ALK inhibitor (crizotinib) showed a higher incidence of SAEs in the urinary system as follows: crizotinib: $1.78 \%$ (95\% CI: $0.77-2.78)$; alectinib: $\quad 0.01 \% \quad(95 \% \quad$ CI: $\quad 0.17-0.20)$; ceritinib: $0.78 \%$ (95\% CI: $0.26-1.81)$; and brigatinib: $0.31 \% \quad(95 \%$ CI: 0.46-1.08). Conversely, the second generation ALK inhibitors, especially ceritinib and brigatinib, showed higher nervous system toxicity as follows: crizotinib: 3.88\% (95\% CI: 2.09-5.66); alectinib: $3.07 \%$ (95\% CI: $0.45-5.70$ ); ceritinib: $8.84 \%$ (95\% CI: $3.93-13.75$ ); and brigatinib: $7.40 \%(95 \%$ CI: $4.70-10.10)$. The forest plots for the SAEs in the five systems are shown in the supplementary files (Figures S4-S8).

\section{The SAEs of the ALK inhibitors}

Figure 3 shows the SAEs arranged by incidence for the 4 ALK inhibitors. The lung toxicity of the ALK inhibitors was observed clearly. The SAE (incidence $>1 \%$ ) comparisons between the two generation of ALK inhibitors are shown in Figure 4. The forest plots for these results are shown in the supplementary files (Figures S9-S46).

For crizotinib, the SAE sequences were arranged by incidence as follows: pneumonia, 4.21\% (95\% CI: 2.13-6.30); thrombotic disease (including pulmonary embolism and venal thrombosis), 3.71\% (95\% CI: 1.85-5.58); pleural effusion, $1.26 \% \quad(95 \% \quad$ CI: $\quad 0.74-1.77)$; pyrexia, $1.14 \% \quad(95 \%$ CI: $0.65-1.64)$; vomiting, $0.60 \%$ (95\% CI: $0.03-1.17)$; 

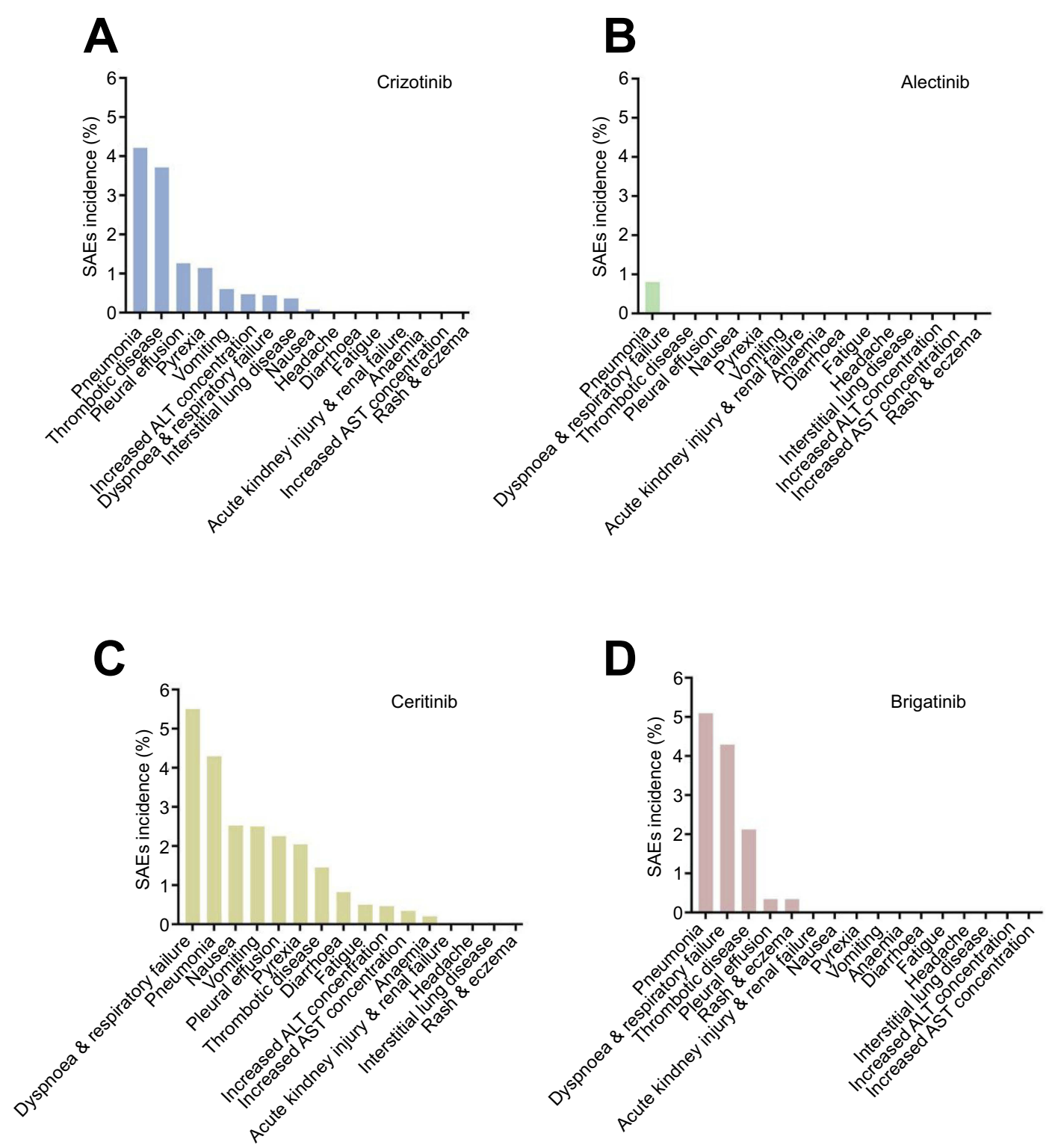

Figure 3 SAEs incidences of two generations ALK inhibitors.

Notes: (A) crizotinib; (B) alectinib; (C) ceritinib; (D) brigatinib.

Abbreviation: ALK, Anaplastic lymphoma kinase.

increased alanine aminotransferase (ALT) concentration, 0.47\% (95\% CI: 0.02-0.96); dyspnea and respiratory failure (including dyspnea, respiratory failure and acute respiratory distress syndrome), $0.44 \%$ (95\% CI: $0.10-0.78)$; ILD, $0.36 \%$ (95\% CI: 0.04-0.77); and nausea, 0.08\% (95\% CI: 0.07-0.22).

Alectinib showed superior safety. The SAE with the highest incidence for alectinib in this meta-analysis was pneumonia (0.80\%, 95\% CI: $0.52-2.11)$. The incidences of the other SAEs for alectinib were less than $0.05 \%$.

Ceritinib showed some drawbacks in terms of safety. The SAE sequence arranged by incidence was as follows: dyspnea and respiratory failure, $5.50 \% \quad(95 \% \quad \mathrm{CI}: \quad 3.10-7.89)$; pneumonia, 4.29\% (95\% CI: 2.79-5.79); nausea, $2.53 \%$ (95\% CI: 1.37-3.69); vomiting, 2.50\% (95\% CI: 0.62-4.38); pleural effusion, $2.26 \%$ (95\% CI: 0.55-3.79); pyrexia, $2.05 \%$ (95\% CI: 0.49-3.61); thrombotic disease, $1.45 \%$ (95\% CI: 0.57-2.34); and diarrhea, 1.45\% (95\% CI: 0.57-2.34).

Brigatinib showed more significant lung toxicity than the other SAEs. The SAE sequence arranged by incidence was as follows: pneumonia, 5.09\% (95\% CI: 2.11-8.07); dyspnea and respiratory failure, $4.29 \%$ (95\% CI: 0.67-7.90); thrombotic disease, 2.13\% (95\% CI: 0.64-3.63); and pleural effusion, $2.26 \%(95 \% \mathrm{CI}$ : $0.55-3.79)$. 


\section{A}

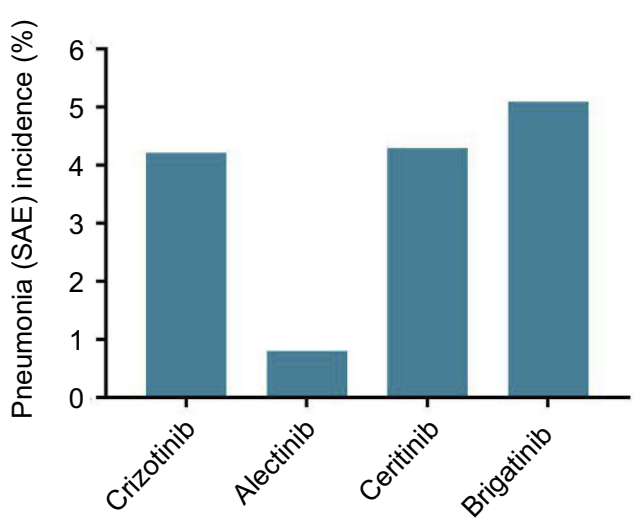

C

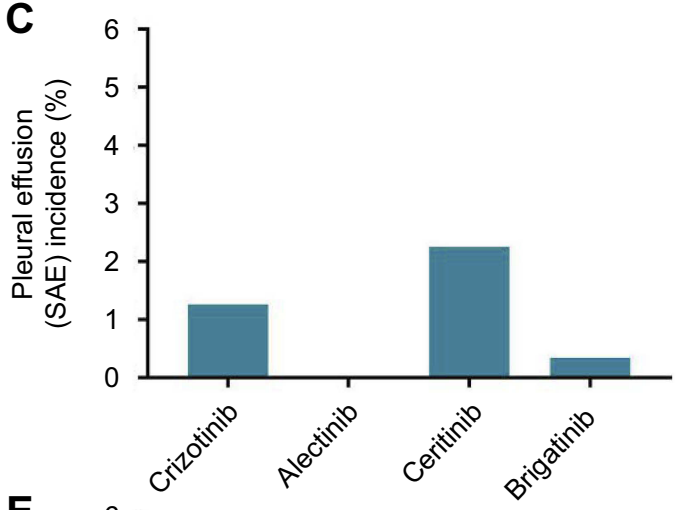

E
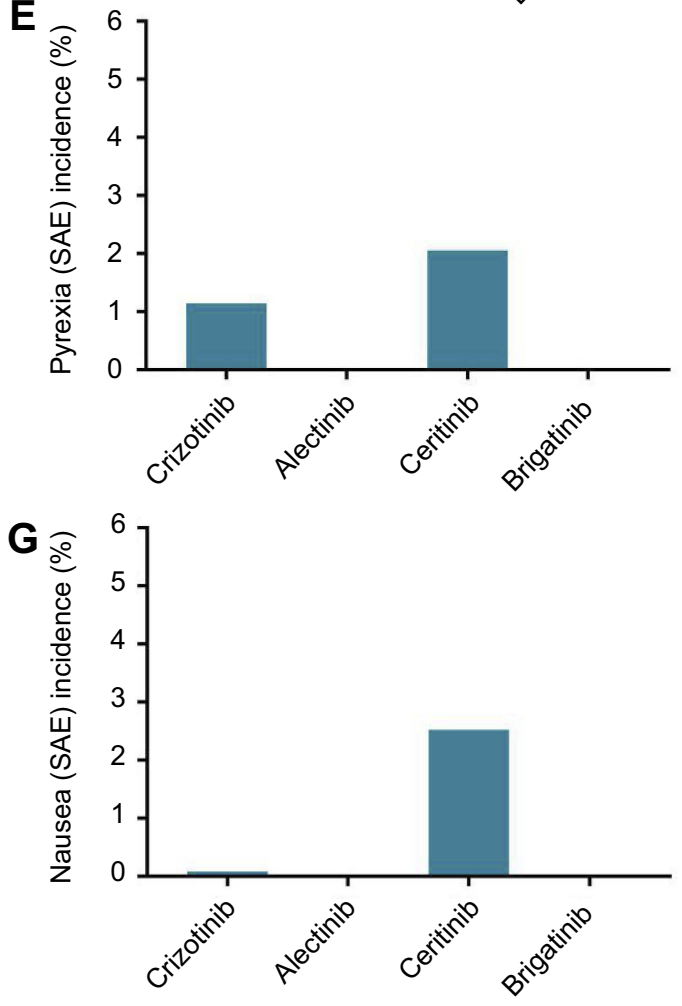

B

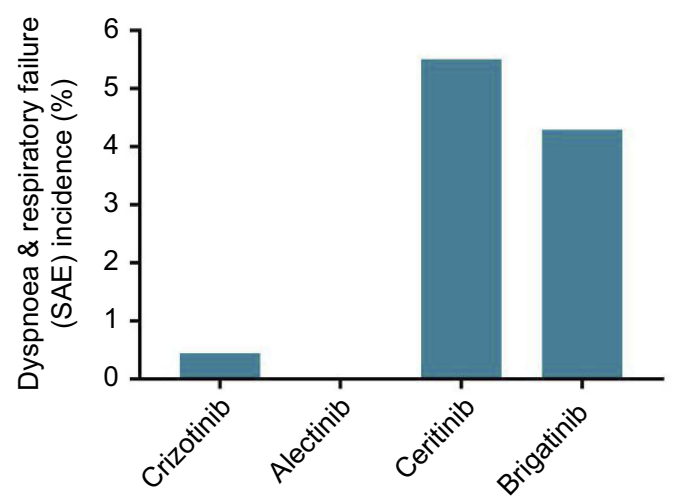

D

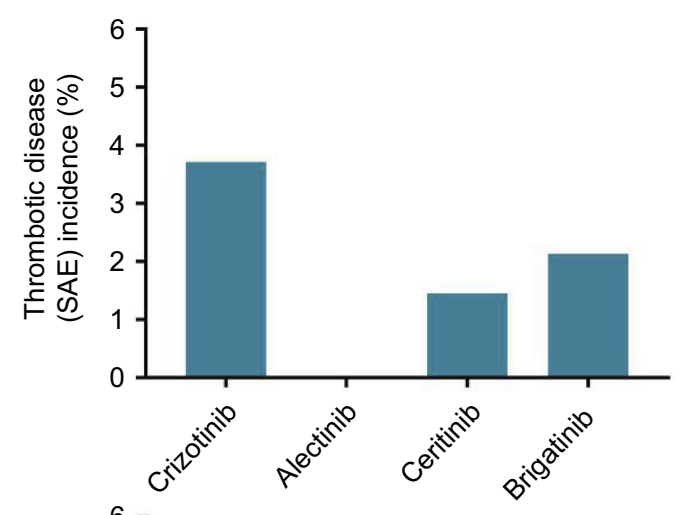

$\mathbf{F}$

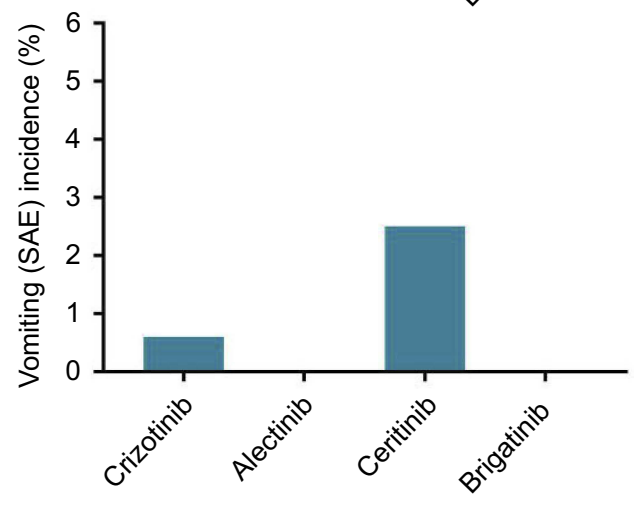

Figure 4 The comparison of SAEs in two generations ALK inhibitors.

Notes: (A) pneumonia; (B) dyspnoea and respiratory failure; (C) pleural effusion; (D) thrombotic disease; (E) pyrexia; (F) vomiting; (G) nausea. Abbreviation: ALK, Anaplastic lymphoma kinase. 


\section{Subgroup analyses}

We set subgroup factors prior to the meta-analysis, including race, treatment lines, driver mutations and ALK inhibitor dosages. When heterogeneity existed in the meta-analysis, subgroup analyses were performed. The subgroup analysis results are shown in Table S3, and the details of the subgroup analyses are shown in the supplementary files. We found that race, treatment lines and driver mutations were important factors for ALK inhibitor treatment.

\section{Publication bias}

Egger's and Begg's tests were performed to evaluate publication bias in this meta-analysis. When atypism occurred between two tests for one index in this meta-analysis, we accepted the results of Egger's test. ${ }^{27}$ The publication bias evaluation results are shown in the supplementary files (Table S4). Most of the results in this meta-analysis had no evidence of publication bias.

\section{Sensitivity analysis}

The sensitivity analysis results showed no significant differences in most of the results in this meta-analysis after omitting any one of the included studies, which indicated that the results of this meta-analysis were robust. Some of the SAE incidences showed poor sensitivity; we considered that this finding was due to the research effect of the small sample size caused by the very low incidence of SAEs. The sensitivity analysis results are shown in the supplementary files (Figures S47-S142)

\section{Discussion}

ALK inhibitors improve the prognosis of ALK-positive NSCLC patients, and most AEs can be well controlled and tolerated. ${ }^{28,29}$ However, the SAEs of ALK inhibitors cannot be ignored clinically. With the wide application of ALK inhibitors, ALK inhibitor-related SAEs have gradually aroused our attention. According to the definition of the ClinicalTrials.gov website, SAEs refer to adverse events that result in death, are life-threatening, require inpatient hospitalization or extend a current hospital stay, result in an ongoing or significant incapacity or interfere substantially with normal life functions, or cause a congenital anomaly or birth defect. Therefore, physicians should pay attention to drug-related SAEs when prescribing ALK inhibitor treatment.

In this meta-analysis, we found that the total AEs of the ALK inhibitors occurred in almost all of the participants and that the total SAEs occurred in more than $20 \%$ of the participants. In particular, SAEs due to ceritinib and brigatinib occurred in more than $40 \%$ of the participants. Alectinib is most likely the safest of the four ALK inhibitor. Regarding the SAE results for the five investigated systems, SAEs of the respiratory system were most common. The second generation ALK inhibitors had a high incidence of SAEs of the nervous system, which was possibly related to their central nervous system (CNS) penetration ability. ${ }^{30}$ In terms of single SAEs, pneumonia seemed to have the highest incidence. In combination with the incidences of plural effusion, dyspnea and respiratory failure, the ALK inhibitors showed significant lung toxicity. ALK inhibitor-related thrombotic disease should draw our attention when providing long-term treatment for elderly, bed-ridden, cancer patients who have a high risk of suffering thrombotic disease. Moreover, ceritinib had high incidences of alimentary SAEs, including nausea, vomiting and diarrhea. Race, the treatment line and the driver mutation seemed to be important factors for ALKrelated SAEs. Generally, the incidences of SAEs increase with the addition of the treatment line. However, alectinib seems to be safer in the second line treatment.

According to the subgroup analysis results, we can draw conclusions regarding the suitability of an ALK inhibitor for a specific population. Based on the subgroup analysis of race, crizotinib may be safer for Asians, especially in terms of the cardiovascular and urinary system SAEs. Alectinib is more suitable for patients with chemotherapy or previous crizotinib treatment in terms of safety, whereas ceritinib is safer as a firstline treatment. Brigatinib showed significant differences in safety between patients with different driver mutations. For patients with ALK mutations or diffusion, treatment with brigatinib is safer.

Admittedly, our meta-analysis has some limitations. Given the low incidences of some SAEs, the small sample size of the research may have influenced the outcomes of this study, resulting in some poor sensitivity evaluation results. Moreover, an insufficient number of clinical trials with results for ALK inhibitors, especially second generation ALK inhibitors, was available for this meta-analysis. Therefore, more clinical trials need to be included to confirm our results.

\section{Conclusion}

ALK-related SAEs should draw attention, especially in terms of lung toxicity. According to this meta-analysis, alectinib seems to be the safest ALK inhibitor. Physicians should focus on related SAEs when prescribing ALK inhibitors. Given that lung cancer patients have poor pulmonary function at baseline, the lung toxicity risk of ALK inhibitors should be evaluated before giving treatment. 


\section{Ethical approval}

All procedures performed in studies involving human participants were in accordance with the ethical standards of the institutional and/or national research committee and with the 1964 Declaration of Helsinki and its later amendments or comparable ethical standards.

\section{Informed consent}

Informed consent was obtained from all individual participants included in the study.

\section{Acknowledgments}

Funding: This work was supported by the Taishan Scholar Foundation of Shandong Province (No. tshw201502061), the Qingdao People's Livelihood Science and Technology Program (16-6-2-3-nsh) and the Qingdao Entrepreneurial Innovation Leading Talent Program.

\section{Disclosure}

The authors report no conflicts of interest in this work.

\section{References}

1. Soda M, Choi YL, Enomoto M, et al. Identification of the transforming EML4-ALK fusion gene in non-small-cell lung cancer. Nature. 2007;448(7153):561-566. doi:10.1038/nature05984

2. Rikova K, Guo A, Zeng Q, et al. Global survey of phosphotyrosine signaling identifies oncogenic kinases in lung cancer. Cell. 2007;131 (6):1190-1203. doi:10.1016/j.cell.2007.11.025

3. Miyanaga A, Shimizu K, Noro R, et al. Activity of EGFRtyrosine kinase and ALK inhibitors for EML4-ALK-rearranged non-smallcell lung cancer harbored coexisting EGFR mutation. BMC Cancer. 2013;13:262-267. doi:10.1186/1471-2407-13-262

4. Hou H, Zhu H, Yan W, et al. Comprehensive molecular characterization of young Chinese patients with lung adenocarcinoma identified a distinctive genetic profile. Oncologist. 2018;23(9):1-8. doi:10.1634/ theoncologist.2017-0629

5. Kwak EL, Bang YJ, Camidge DR, et al. Anaplastic lymphoma kinase inhibition in non-small-cell lung cancer. $N$ Engl J Med. 2010;363 (18):1693-1703. doi:10.1056/NEJMoa1011205

6. Shaw AT, Kim DW, Nakagawa K, et al. Crizotinib versus chemotherapy in advanced ALK-positive lung cancer. $N$ Engl J Med. 2013;368 (25):2385-2394. doi:10.1056/NEJMoa1214886

7. Solomon BJ, Mok T, Kim DW, et al. First-line crizotinib versus chemotherapy in ALK-positive lung cancer. $N$ Engl $J$ Med. 2014;371:2167-2177. doi:10.1056/NEJMoa1410490

8. Pellegrino B, Facchinetti F, Bordi P, Silva M, Gnetti L, Tiseo M. Lung toxicity in non-small cell lung cancer patients exposed to ALK inhibitors: report of a peculiar case and systematic review of the literature. Clin Lung Cancer. 2017;19(2):e151-e161. doi:10.1016/j.cllc.2017.10.008

9. Yamamoto Y, Okamoto I, Otsubo K, et al. Severe acute interstitial lung disease in a patient with anaplastic lymphoma kinase rearrangement-positive non-small cell lung cancer treated with alectinib. Invest New Drugs. 2015;33(5):1148-1150. doi:10.1007/ s10637-015-0284-9
10. Ikeda $\mathrm{S}$, Yoshioka $\mathrm{H}$, Arita $\mathrm{M}$, et al. Interstitial lung disease induced by alectinib (CH5424802/RO5424802). Jpn J Clin Oncol. 2015;45 (2):221-224. doi:10.1093/jjco/hyul83

11. Stang A. Critical evaluation of the Newcastle-Ottawa scale for the assessment of the quality of nonrandomized studies in meta-analyses. Eur J Epidemiol. 2010;25(9):603-605. doi:10.1007/s10654-010-9491-z

12. Higgins JP, Altman DG, Gotzsche PC, et al. The Cochrane Collaboration's tool for assessing risk of bias in randomised trials. BMJ. 2011;343:d5928. doi:10.1136/bmj.d5928

13. Clinicaltrials.gov [homepage on the Internet]. NCT01945021. Study results; 2019 [updated February 15, 2018; cited June 16, 2018]. Available from: https://www.clinicaltrials.gov/ct2/results? cond $=\&$ term=NCT01945021. Accessed April 17, 2019.

14. Peters S, Camidge DR, Shaw AT, et al. Alectinib versus crizotinib in untreated ALK-positive non-small-cell lung cancer. $N$ Engl $J$ Med. 2017;377(9):829-838. doi:10.1056/NEJMoa1704795

15. Lu S, Mok T, Lu Y, et al. Phase 3 study of first-line crizotinib vs pemetrexed-cisplatin/carboplatin in East Asian patients with ALK+ advanced non-squamous non-small cell lung cancer (NSCLC). J Clin Oncol. 2016;34(suppl). doi:10.1200/JCO.2016. 34.15_suppl.9058

16. Blackhall F, Canidge DR, Shaw AT, et al. Final results of the large-scale multinational trial PROFILE 1005: efficacy and safety of crizotinib in previously treated patients with advanced/metastatic ALK-positive non small-cell lung cancer. ESMO Open. 2017;2(3): e000219. doi:10.1136/esmoopen-2017-000219

17. Shaw AT, Gandhi L, Gadgeel S, et al. Phase 2 prospective analysis of alectinib in ALK-positive, crizotinib-resistant non-small-cell lung cancer. Lancet Oncol. 2016;17(2):234-242. doi:10.1016/S14702045(15)00488-X

18. Ou SH, Ahn JS, Petris LD, et al. Alectinib in crizotinib-refractory ALK-rearranged non-small-cell lung cancer: a phase II global study. J Clin Oncol. 2016;34(7):661-668. doi:10.1200/JCO.2015.63.9443

19. Seto T, Kiura K, Nishio M, et al. CH5424802 (RO5424802) for patients with ALK-rearranged advanced non-small-cell lung cancer (AF-001JP study): a single-arm, open-label, phase 1-2 study. Lancet Oncol. 2013;14(7):590-598. doi:10.1016/S1470-2045(13) 70142-6

20. Shaw AT, Kim TM, Crinò L, et al. Ceritinib versus chemotherapy in patients with ALK-rearranged non-small-cell lung cancer previously given chemotherapy and crizotinib (ASCEND-5): a randomised, controlle, open-label, phase 3 trial. Lancet Oncol. 2017;18(7):874-886. doi:10.1016/S1470-2045(17)30072-4

21. Crinò L, Ahn MJ, Marinis FD, et al. Multicenter phase II study of whole-body and intracranial activity with ceritinib in patients with ALK-rearranged non-small-cell lung cancer previously treated with chemotherapy and crizotinib: results from ASCEND-2. J Clin Oncol. 2016;20(34):2866-2873. doi:10.1200/JCO.2015.65.5936

22. Soria JC, Tan DSW, Chiari R, et al. First-line ceritinib versus platinum-based chemotherapy in advanced ALK-rearranged non-small-cell lung cancer (ASCEND-4): a randomised, open-label, phase 3 study. Lancet Oncol. 2017;389(10072):917-929. doi:10.1016/S0140-6736(17)30123-X

23. Kim DW, Mehra R, Tan DSW, et al. Activity and safety of ceritinib in patients with ALK -rearranged non-small-cell lung cancer (ASCEND-1): updated results from the multicentre, open-label, phase 1 trial. Lancet Oncol. 2016;17(4):452-463. doi:10.1016/ S1470-2045(15)00614-2

24. Nishio M, Murakami H, Horiike A, et al. Phase I study of ceritinib (LDK378) in Japanese patients with advanced, anaplastic lymphoma kinase-rearranged non-small-cell lung cancer or other tumors. J Clin Oncol. 2015;10(7):1058-1066.

25. Camidge DR, Kim DW, Tiseo M, et al. Exploratory analysis of brigatinib activity in patients with anaplastic lymphoma kinase-positive non-smallcell lung cancer and brain metastases in two clinical trials. J Clin Oncol. 2018;36(26):2693-2701. doi:10.1200/JCO.2017.77.5841 
26. Gettinger SN, Bazhenova LA, Langer CJ, et al. Activity and safety of brigatinib in ALK-rearranged non-small-cell lung cancer and other malignancies: a single-arm, open-label, phase 1/2 trial. Lancet Oncol. 2016;17(12):1683-1696. doi:10.1016/S1470-2045(16)30392-8

27. Hayashino Y, Noguchi Y, Fukui T. Systematic evaluation and comparison of statistical tests for publication bias. J Epidemiol. 2005;15 (6):235. doi:10.2188/jea.15.235

28. Costa RB, Costa RLB, Talamantes SM, et al. Systematic review and meta-analysis of selected toxicities of approved ALK inhibitors in metastatic non-small cell lung cancer. Oncotarget. 2018;9 (31):22137-22146. doi:10.18632/oncotarget.25154
29. Zhu Q, Hu H, Jiang F, et al. Meta-analysis of incidence and risk of severe adverse events and fatal adverse events with crizotinib monotherapy in patients with ALK-positive NSCLC. Oncotarget. 2017;8(43):7537275380 .

30. Gadgeel SM, Gandhi L, Riely GJ, et al. Safety and activity of alectinib against systemic disease and brain metastases in patients with crizotinib-resistant ALK-rearranged non-small-cell lung cancer (AF-002JG): resultsfrom the dose-finding portion of a phase 1/ 2 study. Lancet Oncol. 2014;15(10):1119-1128. doi:10.1016/ S1470-2045(13)70510-2

\section{Publish your work in this journal}

Cancer Management and Research is an international, peer-reviewed open access journal focusing on cancer research and the optimal use of preventative and integrated treatment interventions to achieve improved outcomes, enhanced survival and quality of life for the cancer patient.
The manuscript management system is completely online and includes a very quick and fair peer-review system, which is all easy to use. Visit http://www.dovepress.com/testimonials.php to read real quotes from published authors. 